\title{
Sprachalltag II: Sprachatlas - Digitalisierung - Nachhaltigkeit und das Arno-Ruoff-Archiv am Ludwig-Uhland-Institut für Empirische Kulturwissenschaft der Universität Tübingen. Ein Projektbericht
}

\author{
Rudolf Bühler (Tübingen)
}

\begin{abstract}
Since 2015, the project Sprachalltag II has been running at the Institute of Historical and Cultural Anthropology in Tübingen. In addition to the final processing of the Sprachatlas von Nord Baden-Württemberg (SNBW), begun in 2009, and the creation of a popular, online Sprechender Sprachatlas von Baden-Württemberg, the goal is to research and digitize the extensive material of the Arno-Ruoff archive for further linguistic and ethnologic studies.

This project in particular includes the transcription and alignment of the dialect recordings collected by Ruoff and Bausinger since 1955 for the so called Zwirner corpus as well as the publication of the edited texts in a database in cooperation with the IDS Mannheim. After researching mainly on the morphological and syntactic level of the corpus, the project now also enables phonological examinations of the spoken language throughout the federal state of Baden-Württemberg and the area of Bavarian Swabia via the database.

This report will introduce the nature and extent of the hitherto edited recordings in more detail and show how, in the sense of a cross-disciplinary collaboration, Empirical Cultural Studies can benefit from the content development of the Tübingen corpus. In the course of editing, the transcripted dialect recordings are assigned to thematic categories by using keywords such as leisure or modernization. This includes the means of enquiry of the corpus to a content-related level that can serve the research fields of Historical and Cultural Anthropology.
\end{abstract}

\section{$1 \quad$ Einleitung}

Am Ludwig-Uhland-Institut für Empirische Kulturwissenschaft der Universität Tübingen (LUI) läuft seit 2015 unter der Leitung von Hubert Klausmann und Reinhard Johler das Projekt Sprachalltag II, das neben der abschließenden Bearbeitung des 2009 begonnenen Sprachatlas von Nord Baden-Württemberg (SNBW) und der Erstellung eines populärwissenschaftlichen, online verfügbaren Sprechenden Sprachatlas von Baden-Württemberg die Aufgabe hat, das umfangreiche Material des Arno-Ruoff-Archivs ${ }^{1}$ zu erschließen und zu digitalisieren.

\footnotetext{
${ }^{1}$ Für ein Verzeichnis der Aufnahmen cf. Ruoff 1973: 275-384. 
Wie bereits im Vorgängerprojekt Sprachalltag I ist es das Ziel, sprach- und kulturwissenschaftliche Forschungsinteressen zu berücksichtigen. Zum einen werden durch traditionelle Erhebungsmethoden gewonnene Daten dialektgeographisch ausgewertet, um die letzte Forschungslücke bei den Kleinraumatlanten im Südwesten des deutschen Sprachgebiets zu schließen (cf. Bühler 2013). Zum anderen sind subjektive Angaben zur Sprache und freie Rede der Mundartsprecherinnen und Mundartsprecher Gegenstand unserer Forschung (cf. Bürkle 2014, Leonhardt 2015, Ruoff 1973: 42-68). Zu beiden Aspekten sind in der neustrukturierten Arbeitsstelle Sprache in Südwestdeutschland, die im Rahmen der Alemannentagung 2014 in Tübingen als Fortsetzung der vormaligen Tübinger Arbeitsstelle „Sprache in Südwestdeutschland“ (cf. Ruoff 1997) eröffnet wurde, umfangreiche Datenkorpora untergebracht. Dazu gehören die etwa 200 Fragebücher und die erhebungsbegleitend mitgeschnittenen Tonaufnahmen zum SNBW und zum Sprechenden Sprachatlas von Baden-Württemberg aus den Jahren 2009-2017 sowie die von Ruoff und Bausinger gesammelten etwa 2.000 Tonaufnahmen, hauptsächlich aus den Jahren 1955-1968, nebst den 23.800 maschinenschriftlichen Seiten zeitgenössischer Transkripte.

Die schrittweise Auswertung der für den $S N B W$ angelegten Fragebücher, die Bereitstellung der dialektgeographischen Forschungsergebnisse in Baden-Württemberg für eine interessierte Öffentlichkeit im Rahmen eines Sprechenden Sprachatlas sowie die Einbindung der von Arno Ruoff und Hermann Bausinger seit 1955 angefertigten Tonaufnahmen in eine fächerübergreifend verwendbare Datenbank bilden nun den Kern unserer Forschungsarbeit, die als Ausgangspunkt für zukünftige linguistische und kulturwissenschaftliche Untersuchungen zur gesprochenen Sprache in Südwestdeutschland dient. Das durch das Ministerium für Ländlichen Raum und Verbraucherschutz Baden-Württemberg geförderte Projekt Kultureller Wandel im Ländlichen Raum mit einer Laufzeit vom 01.08.2018 - 31.12.2019 nutzt bereits die neu geschaffenen Recherchemöglichkeiten der Datenbank, um anhand der thematischen Bereiche „Leben und Arbeiten“, „Freizeit und Infrastruktur“ und „Medizinische Versorgung“ Aspekte des kulturellen Wandels des ländlichen Raumes in Baden-Württemberg darzustellen. ${ }^{2}$

\section{Die Teilprojekte von Sprachalltag II}

Sprachalltag II besteht aus drei Teilprojekten:

1. Der Sprachatlas von Nord Baden-Württemberg (SNBW)

2. Der Sprechende Sprachatlas von Baden-Württemberg

3. Die Digitalisierung des Arno-Ruoff-Archivs

\subsection{Der Sprachatlas von Nord Baden-Württemberg (SNBW)}

In den Jahren 2009-2014 wurden im Rahmen des Vorgängerprojektes Sprachalltag I (cf. Bühler 2013) in etwa 170 Erhebungsorten im Norden von Baden-Württemberg (siehe Abbildung 1) Mundartaufnahmen für den $S N B W$ durchgeführt und ein erster Kartenband mit 67 Karten zum Kurzvokalismus fertiggestellt (Klausmann 2015).

\footnotetext{
2 Projektbeschreibung unter LUI (2019b).
} 
In der Folge konnte der zweite Band bearbeitet werden, der ca. 90 Karten zum Langvokalismus, zu den Diphthongen, zu Vokalquantitäten und zum Konsonantismus enthält (Klausmann 2016).

Die ersten beiden Bände wurden in jeweils vier Kartenschnitten angefertigt, die für jedes Kartenthema den rhein- und südfränkischen Westen, den ostfränkischen Nordosten, das schwäbisch-fränkische Zentrum und den ostschwäbischen Südosten des Untersuchungsgebiets (UG) behandeln (siehe Abbildung 2). Die Aufteilung der Karten ergab sich aus der zum damaligen Zeitpunkt unsicheren Projektlaufzeit, sodass ein Teilgebiet etwa im Rahmen einer Dissertation hätte bewältigt werden können. Auch deshalb sind die Karten dieser ersten beiden Bände mit dem Grafikprogramm CorelDraw sozusagen noch „handgezeichnet“" worden.

Die erhobenen Sprachdaten wurden also zunächst nicht in eine Datenbank eingegeben, sondern die Karten ohne Umweg direkt aus den Fragebüchern heraus angefertigt. Die beiden ersten Bände sind heute über die UB-Kataloge in Tübingen oder Freiburg als e-ressource erreichbar und stehen dort als pdf-Dateien zum Download bereit (Klausmann 2015, 2016).

Der dritte Kartenband beschäftigt sich mit den grammatischen Problemen im UG und enthält 105 Karten hauptsächlich zur Morphologie der Verben, aber auch zu den Pronomina und zur Flexion der Substantive. Diese Karten entstanden nun in Kooperation mit dem eScience-Center der Uni Tübingen, das als einen Schwerpunkt seiner Arbeit die Unterstützung der Digital Humanities hat und die Bereitstellung der dazugehörigen Infrastrukturen bietet.

Die Karten für den $S N B W$ entstehen seit dem dritten Band mit der Geo-Suite QGIS, die auf eine PostgreSQL-Datenbank mit der Erweiterung OpenGIS aufsetzt. Auch die Karten der ersten beiden Bände konnten inzwischen in diese Umgebung übertragen werden, sodass nun ein einheitliches, technisch aktuelles Publikationsformat der insgesamt fünf Bände des $S N B W$ vorliegt.

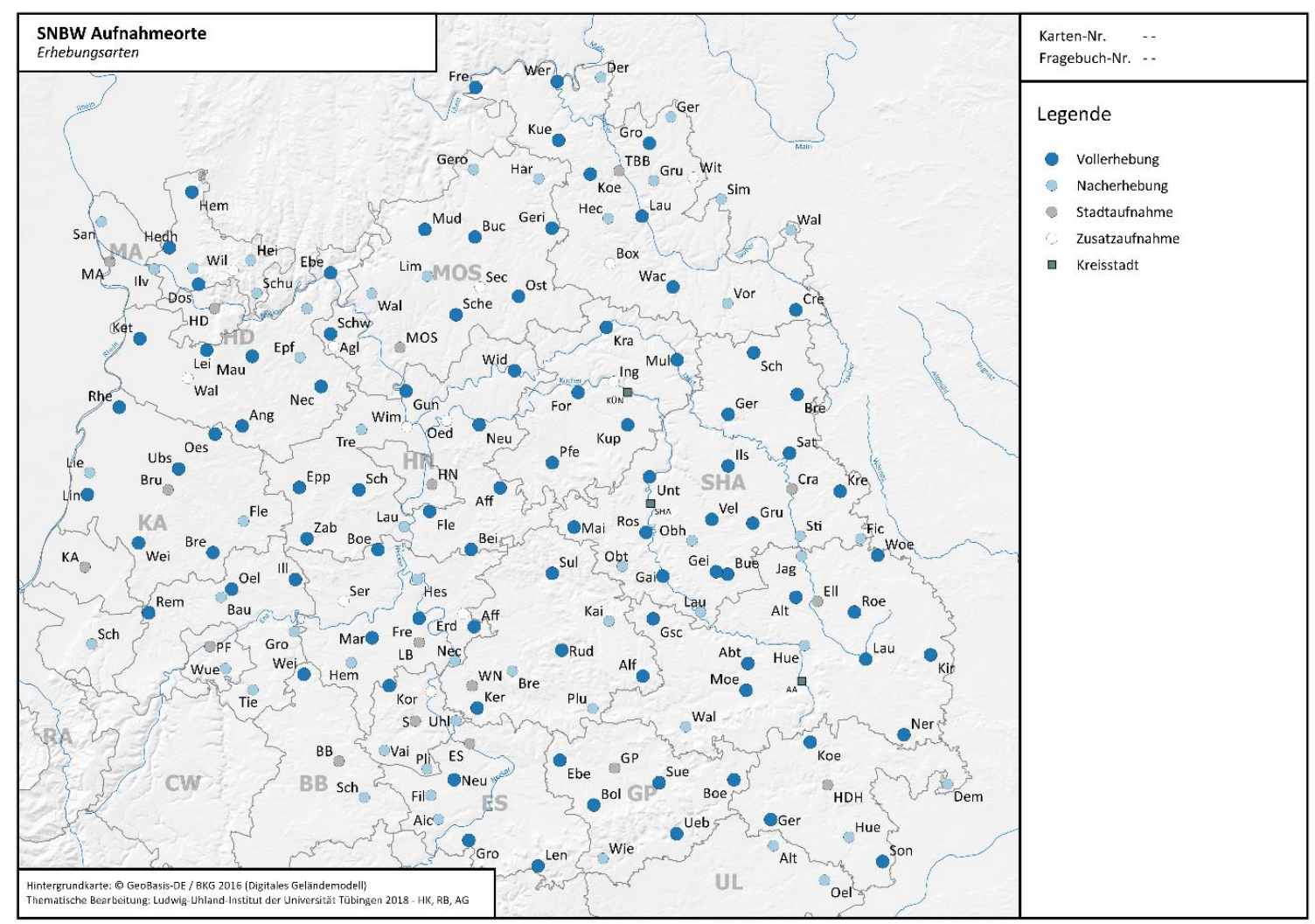

Abbildung 1: Das Untersuchungsgebiet des $S N B W$ 

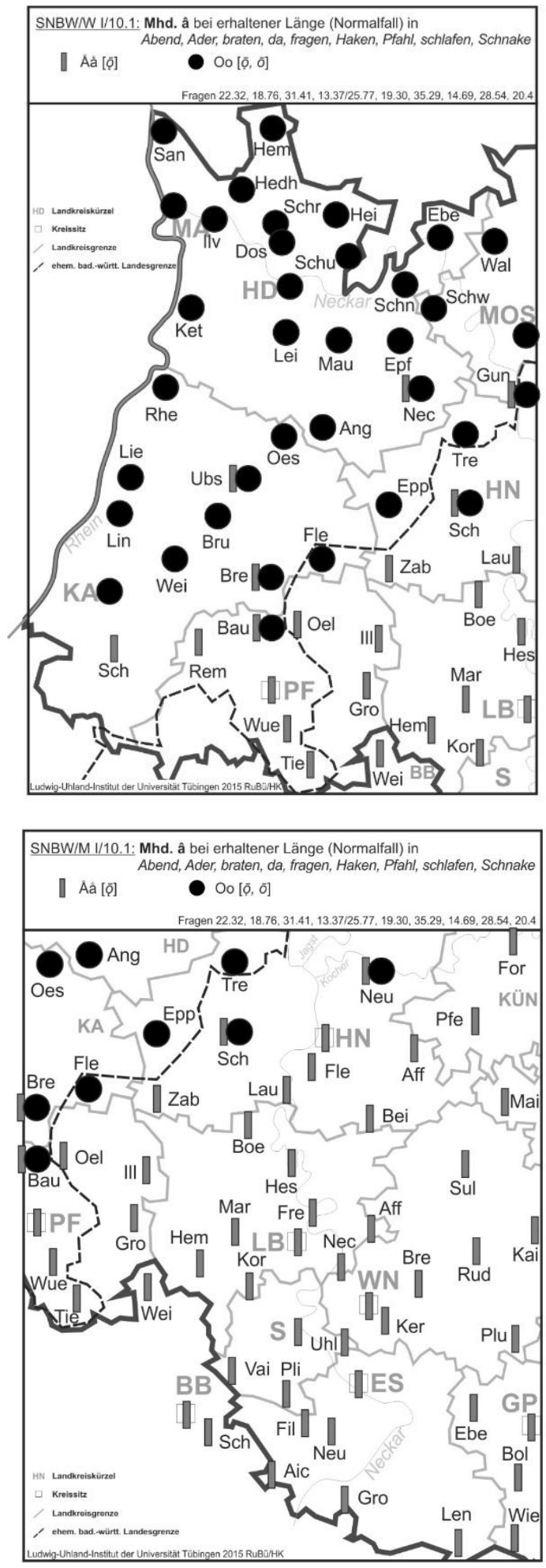
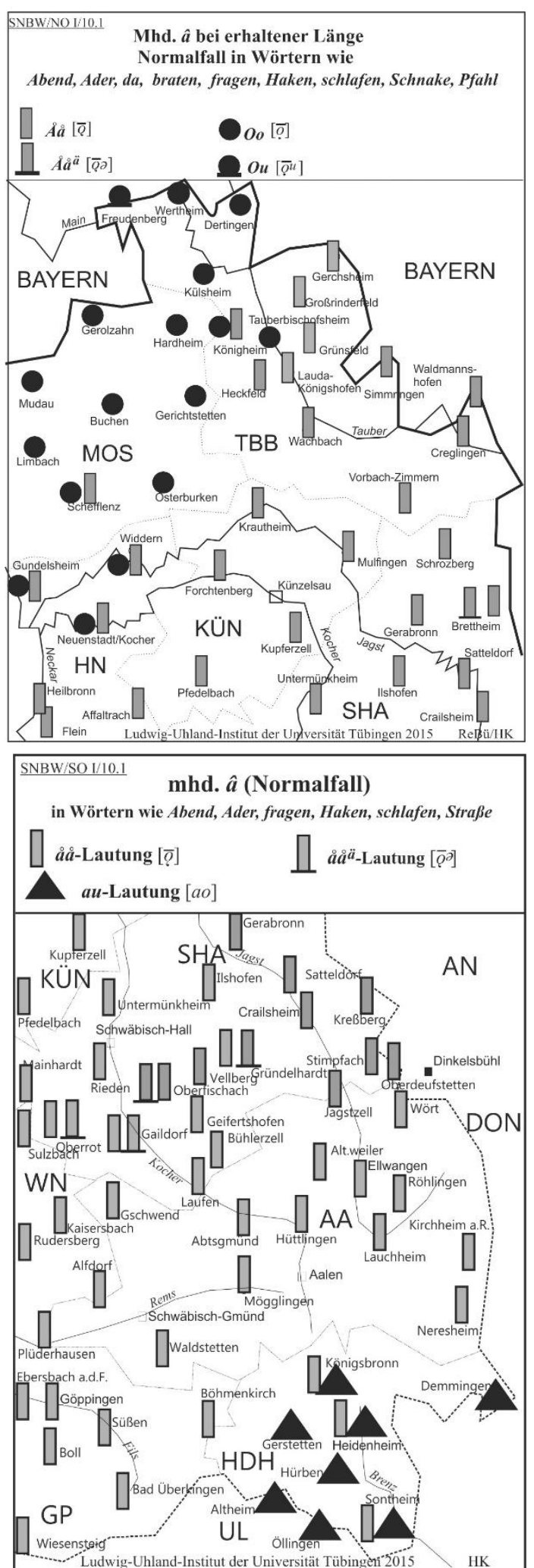

Abbildung 2: Die Kartenschnitte des $\mathrm{SNBW}$ 


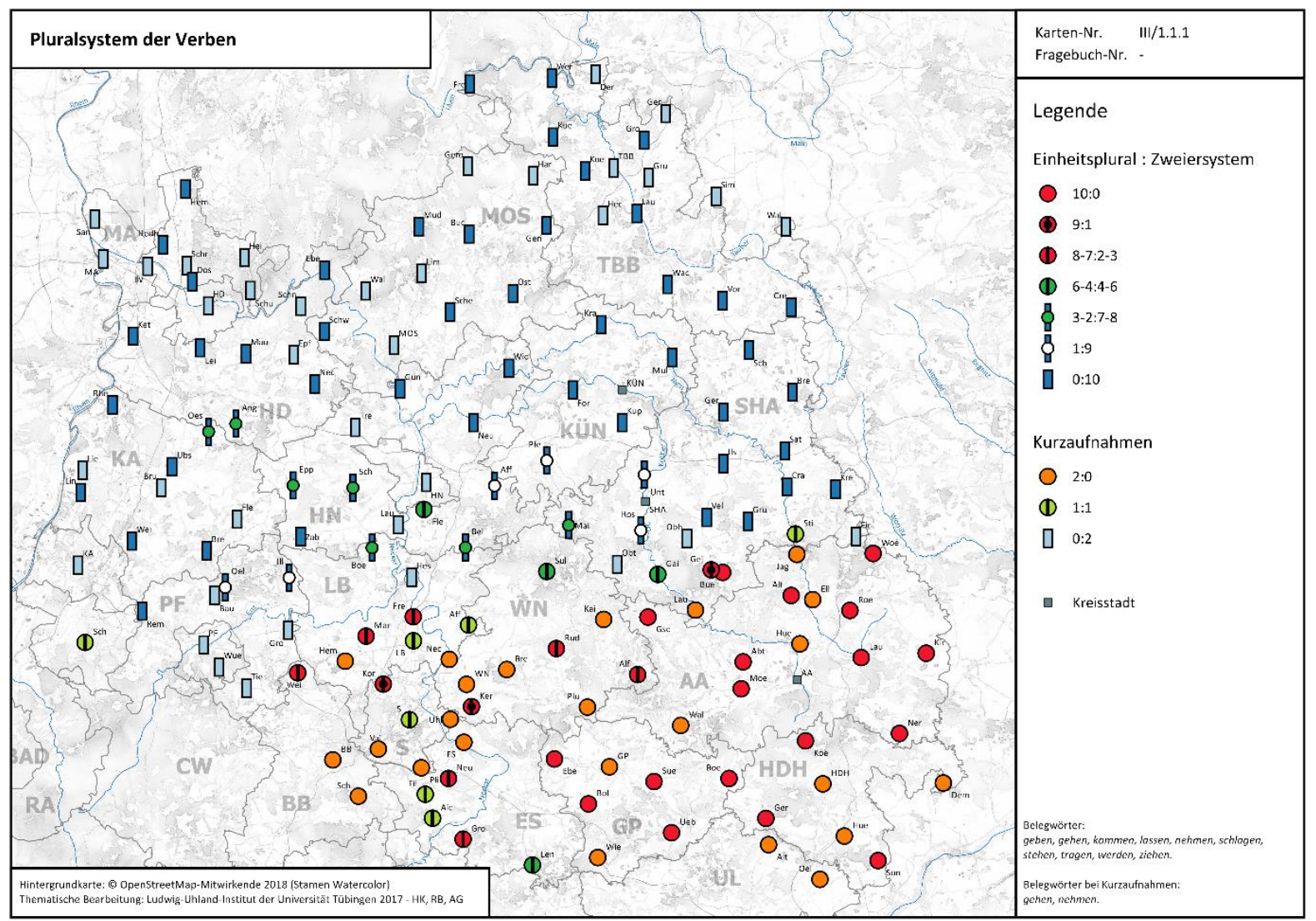

Abbildung 3: Karte aus dem dritten Band des $S N B W$ mit georeferenzierten Ortspunkten

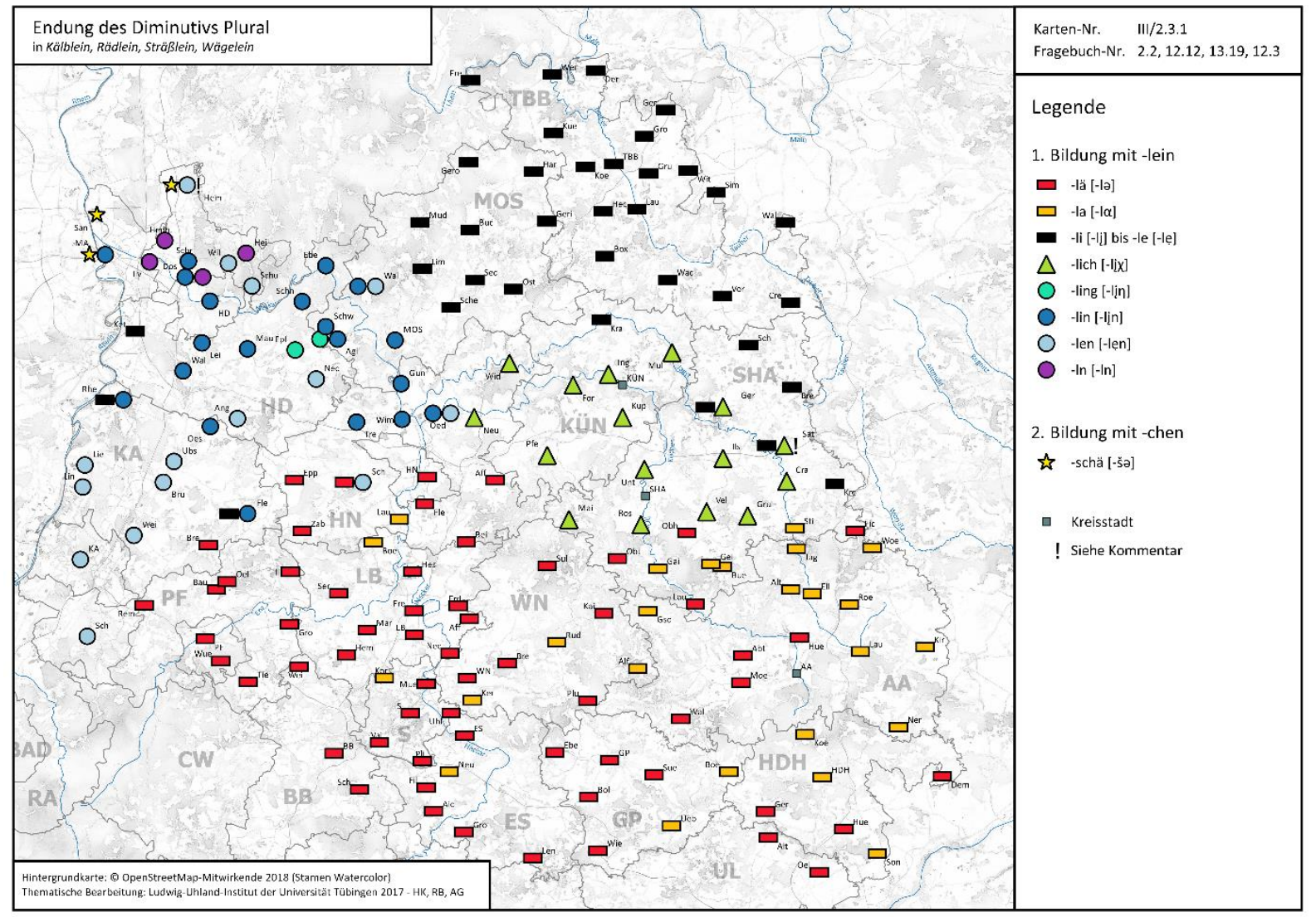

Abbildung 4: Verwendung farbiger Symbole auf den Karten des $S N B W$ zur verbesserten Übersicht 
Das neue Format stattet die Karten jetzt mit georeferenzierten Ortspunkten aus, und erstmals kommen hier auch farbige Symbole zum Einsatz, die zu einer verbesserten Übersichtlichkeit des Kartenbildes beitragen (siehe Abbildungen 3, 4). Eine größere Anzahl von Kodierungsmöglichkeiten schafft einen niedrigeren Abstraktionsgrad, der ein Erfassen der dargestellten sprachlichen Verhältnisse stark vereinfacht. Das gesamte UG erscheint jetzt auf einem einzigen Kartenbild, das jeweils, wie beim benachbarten Südwestdeutschen Sprachatlas (SSA) (Gabriel et al. 1989-2011), mit einem Relief unterlegt ist.

Da bei dem verwendeten Kartenprogramm auf den Legenden oft nicht genügend Raum für erläuternde Begleittexte vorhanden war, wurden von Hubert Klausmann für alle Karten des dritten Bandes bereits eigene Kommentare verfasst. Die Bearbeitung dieses Kartenbandes stand zum Zeitpunkt der Tagung kurz vor dem Abschluss, und die Publikation der Karten und der dazugehörigen Kommentare erfolgte Ende 2017 (Klausmann 2017a, 2017b).

Die Bände 4 und 5 des $S N B W$ sind in den Jahren 2017 und 2018 bearbeitet worden. Darin werden die Themen Landwirtschaft, Wald- und Holzwirtschaft, freilebende Tiere, Wetter, Obst und Gemüse, Einzäunungen, Haus und Stall, der Mensch, das Backen, Küche und Wohnung, Essen und Trinken, Familie und Verwandtschaft, Menschliche Gemeinschaft, die Zeiteinteilung sowie Richtungs-, Zeit- und Ortsadverbien auf 102 bzw. 119 Karten behandelt. Zu den Bänden 4 und 5 sowie zu den ersten beiden Bänden des $S N B W$ werden ebenfalls erläuternde Kommentare erstellt und voraussichtlich Ende 2019 publiziert.

Die Kartenbände 3-5 stehen wie die überarbeiteten ersten beiden Lieferungen des $S N B W$ über unsere Homepage (LUI 2019a) oder im Katalog der Universitätsbibliothek Tübingen zum Download zur Verfügung (Klausmann 2018a, 2018b, 2018c, 2019a, 2019b).

Damit steht der $S N B W$ im Jahr 2019 abschließend bei 485 publizierten Karten zur Phonologie, Morphologie sowie zur Lexik und wird in Zukunft die Möglichkeit bieten, ,,diachrone Betrachtungen auch für den Norden von Baden-Württemberg anzustellen um sprachgeographische Bewegungen, Entwicklungen und deren Antrieb erkennbar zu machen“ (Bühler 2014: 245). Frühere Forschergenerationen (z. B. Haag 1929/30, Moser 1954/55, Baur 1967, Ruoff 1967, 1992) haben von ,der durch die Tradition württembergischer Dialektologie gebotenen Möglichkeit, durch den Vergleich früherer Mundartbeschreibungen mit neuem Sprachstand Sprachentwicklung zu untersuchen und kartographisch darstellen zu können [...] gelegentlich Gebrauch gemacht" (Ruoff 1982: 134, cf. Bühler 2015: 28). Für die Südhälfte des Landes BadenWürttemberg wurde der SSA in der Vergangenheit bereits ,,als Forschungsinstrument und Ausgangspunkt für vergleichende Detailstudien“" (Schrambke 2009: 67) genutzt (z. B. Streck 2012, Schwarz 2015). Genauere Untersuchungen ausgehend von aktuellen Sprachatlasdaten sind nun auch für den Norden im Gebiet des $S N B W$ möglich (z. B. Bühler 2016). Auch besteht nun die Chance zur genauen Charakterisierung des Schwäbischen bzw. Rhein-, Süd- oder Ostfränkischen, indem auch grammatische oder lexikalische Kriterien für die Einteilung der Mundarten im Norden Baden-Württembergs herangezogen werden können.

\subsection{Der Sprechende Sprachatlas von Baden-Württemberg}

Mit der Erfahrung aus der Arbeit am $S N B W$ war der Wunsch entstanden, über einen online verfügbaren populärwissenschaftlichen Sprechenden Sprachatlas die bisher aus $S N B W$ und 
SSA gewonnenen dialektgeographischen Erkenntnisse aus ganz Baden-Württemberg (siehe oben) sowie ergänzendes Tonmaterial, einer breiten Öffentlichkeit zugänglich zu machen. $S N B W$ und $S S A$ pflegen damit nicht nur die „Erinnerung an die substanziellen Großprojekte der Sprachforschung in der Region“, sondern reaktivieren „die regionalen Traditionen der Sprachwissenschaft zur Begründung neu zu setzender Aktivitäten“ (Tschofen/Keller-Drescher 2009: 13). Mit dem Sprechenden Sprachatlas von Bayern (SBS 2013) besteht bereits ein Modell für den benachbarten Freistaat. Mit einem komprimierten Fragebuch, das in 60-70 Minuten für einen Ort bearbeitet werden kann, wurden für Aufnahmeorte im gesamten Land Audio-Vorlagen erstellt. Sie sind heute als Datenkorpus Teil des Arno-Ruoff-Archivs.

Das Ortsnetz war so gewählt worden, dass pro Landkreis zumindest eine Erhebung durchgeführt werden konnte. Die dialektgeographischen Informationen zu jedem Lemma sind den Karten und der Datenbank des SSA für den Süden von Baden-Württemberg, den Fragebüchern des $S N B W$ für den Norden des Landes entnommen. Sie erscheinen als farbige Flächen auf der Karte, sodass die Betrachterinnen und Betrachter die dargestellten sprachlichen Verhältnisse rasch erfassen können. Die Farbgebung lässt sich über eine in Laienschreibweise gehaltene Legende aufschlüsseln (siehe Abbildung 5). Ein Mehrwert des Sprechenden Sprachatlas besteht nun auch darin, dass erstmals Sprachkarten von ganz Baden-Württemberg in einem größeren Umfang zur Verfügung stehen.

Mit den dialektgeographischen Informationen erhalten die Benutzerinnen und Benutzer ein Layer mit dem eigentlichen Sprach-Tool. Die Darstellung in der Benutzeroberfläche erfolgt durch die Open-Source JavaScript Bibliothek Leaflet, welche die meisten Desktop- und MobilBrowser unterstützt. Die abgebildeten Play-Buttons animieren zum Klicken, und die ausgeschnittenen Tonbelege zur kartierten Lautung werden hörbar. $\mathrm{Zu}$ den Karten bekommen die Userinnen und User jeweils auch einen kurzen Kommentar, der die lautlichen Verhältnisse erläutert und beispielsweise einfache etymologische Hinweise zu den Ausdruckstypen enthält. Darüber hinaus werden auf dieser Plattform Ausschnitte aus den Tonaufnahmen des ArnoRuoff-Archivs von jeweils etwa einer Minute Länge präsentiert, welche die Benutzerinnen und Benutzer dann ebenfalls über georeferenzierte Play-Symbole auf der Karte aufrufen können.

Eine Vorab-Version dieses Sprechenden Sprachatlas mit 60 Karten und 37 anklickbaren Aufnahmeorten war ab Herbst 2016 über unsere Homepage (LUI 2019a) zu erreichen. Dieser Prototyp sowie eine Karte mit Mundart-Proben aus etwa 20 Orten des gesamten schwäbischen Sprachraumes konnte schon bei der großen Landesausstellung „Die Schwaben - Zwischen Mythos und Marke“ im Württembergischen Landesmuseum Stuttgart (22.10.2016-23.4.2017) präsentiert werden. Diese Pilotfassung hat als Station bei der Schwabenausstellung in Stuttgart große Beachtung bei den Besucherinnen und Besuchern gefunden. Der Atlas ist nun fertiggestellt, umfasst über 100 Karten, bis zu 57 Audiopunkte je Karte und wurde im Frühjahr 2018 der Öffentlichkeit präsentiert. 


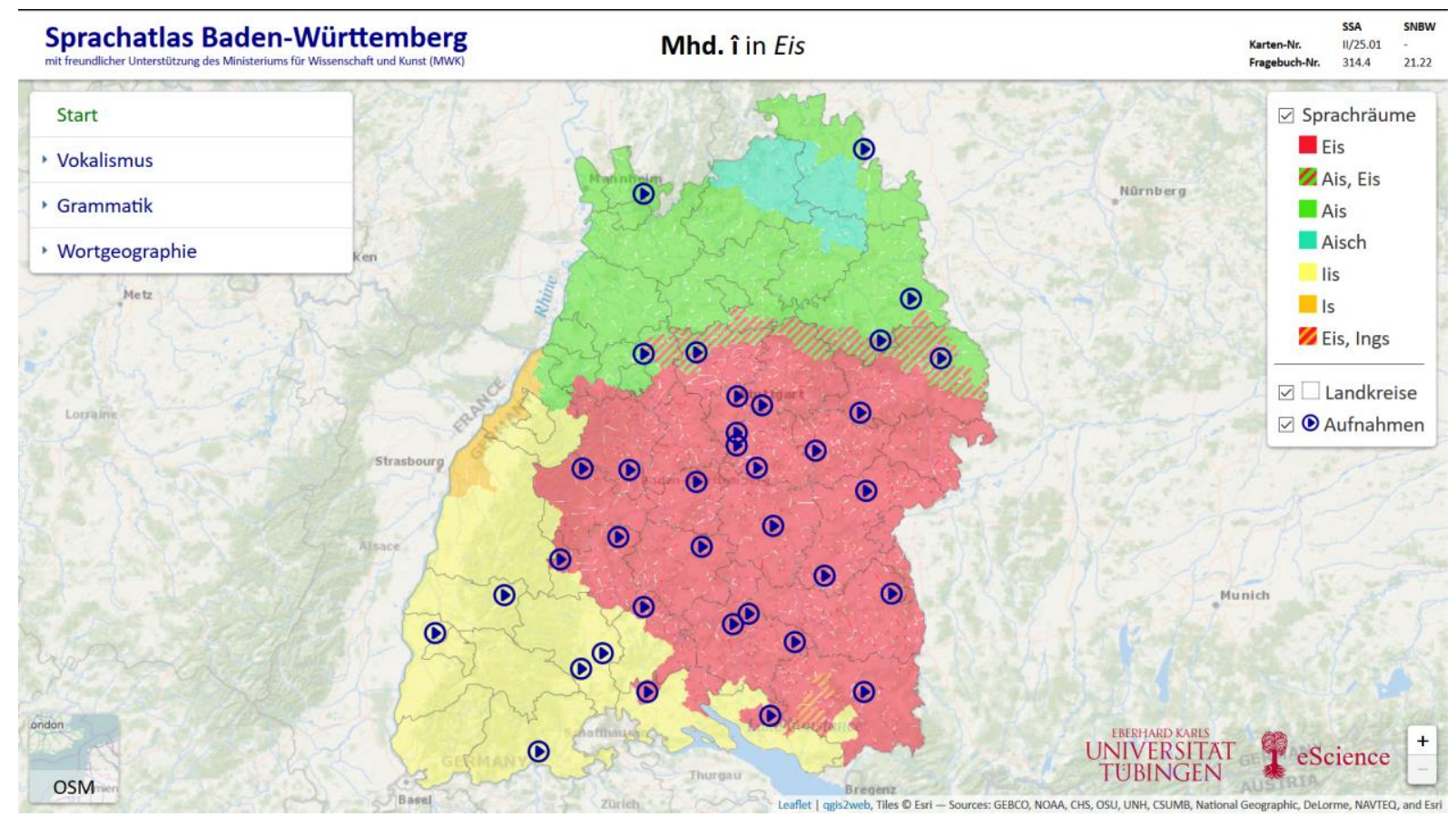

Abbildung 5: Lautgeographische Karte des Sprechenden Sprachatlas von Baden-Württemberg (LUI 2019a)

\subsection{Die Digitalisierung des Arno-Ruoff-Archivs}

Als Kern des gesamten Projekts kann man die Digitalisierung des Arno-Ruoff-Archivs bezeichnen. Im Jahr 1955 hatten Arno Ruoff und Hermann Bausinger damit begonnen, zunächst für das sogenannte Zwirner-Korpus (cf. Zwirner 1964, Ruoff 1965: 94), dann für weitere Projekte, mundartliche Tonaufnahmen in Baden-Württemberg, Bayerisch-Schwaben, Vorarlberg und Liechtenstein anzufertigen (siehe Abbildung 6, Ruoff 1973: 283-385). In den durchschnittlich etwa zehn bis 15 Minuten dauernden Aufnahmen erzählen die Mundartsprecherinnen und Mundartsprecher in freier Rede zu verschiedenen Themen wie Arbeitsabläufen in Handwerk und Landwirtschaft, Festen und Bräuchen im Ort oder Kriegserlebnissen. Sie geben Auskunft über die Alltagsverhältnisse ihrer meist ländlich geprägten Lebenswelt und liefern einen kulturellen Querschnitt ihrer erzählten Zeit, die meist in der ersten Hälfte des 20. Jahrhunderts liegt (cf. Ruoff 1973: 84-87). Die Sammlung des Archivs umfasst über 2.000 Aufnahmen mit 23.800 (siehe oben) literarisch oder phonetisch transkribierten Textseiten (cf. Ruoff 1973 123-145). Zahlreiche dieser Transkripte finden sich in Publikationen der Phonai-Reihe des IDS in Mannheim sowie in den Bänden Alltagstexte I und II der Tübinger Idiomatica-Reihe (Pfeffer/Lohnes 1984; Ruoff 1984, 1985). Hier erschienen auch mehrere Arbeiten, die am Textkorpus Untersuchungen zu vornehmlich morphologischen Themen durchgeführt haben (Eisenmann 1973, Graf 1977, Stadelmann 1978, Gersbach/Graf 1984, 1985, Mikosch 1987, Vahl-Seyfarth 1987, Löffelad 1989). Inhaltlich sind diese Texte jedoch noch nicht untersucht worden, wobei der mittlerweile entstandene zeitliche Abstand zwischen Forschenden und Quelle diese Perspektive immer interessanter werden lässt.

Zunächst boten sich die in Alltagstexte I und II veröffentlichten literarischen Transkripte (Ruoff 1984, 1985) als Experimentierfeld an. Mithilfe von OCR-Scannern sollten die gedruckten Texte digitalisiert und maschinenlesbar gemacht werden. Die Art der literarischen Transkription mit 
den zahlreichen diakritischen Zeichen (cf. Ruoff 1984: XIV-XVI, Schallert 2014: 130) sowie eine Vielzahl handschriftlicher Eintragungen in die literarischen Transkripte der unveröffentlichten Aufnahmen verursachten den verschiedenen getesteten OCR-Programmen jedoch ungeahnte Probleme. Auch war die Durchsuchbarkeit der digitalisierten Texte nach Stichwörtern in der literarischen Form nicht gegeben. Nach ausführlichen Beratungen mit den Kolleginnen und Kollegen am Deutschen Seminar der Uni Zürich um Elvira Glaser, wo ebenfalls an der Transkription dialektaler Sprachaufnahmen gearbeitet wird, sowie mit dem IDS in Mannheim, wo vom dortigen Leiter des Programmbereichs „Mündliche Korpora“ Thomas Schmidt das Werkzeug zur Annotation mündlicher Daten EXMARaLDA (Schmidt 2019) entwickelt wurde, entschieden wir uns für eine standarddeutsche Transkriptionsweise (cf. Ruoff 1973: §91). Diese erlaubt neben der linguistischen auch eine inhaltliche, kulturwissenschaftlich motivierte Auswertung der bearbeiteten Texte ,,als wichtiger Bestandteil einer ,Oral History“ Baden-Württembergs“, der „Aufschluss über Lebensverhältnisse, Alltagserfahrungen und subjektive Deutungsmuster der historischen AkteurInnen geben“3 kann. Seit 2016 besteht ein Kooperationsvertrag zwischen dem IDS und dem LUI. So erfolgen nun im EXMARaLDA-Partitur-Editor Transkription, Alignierung und Annotation der in Mannheim gespeicherten und dem LUI von dort dankeswerterweise zur Verfügung gestellten Digitalisate aus dem Korpus des Arno-Ruoff-Archivs.

Für die Alignierung von Tondateien und standarddeutschen Umschriften kamen zunächst die Interviews aus Baden-Württemberg und Bayerisch-Schwaben in Frage. Das Kernkorpus der im Rahmen von Sprachalltag II bearbeiteten Aufnahmen umfasst ca. 800 Dateien, von denen im Jahr 2017 über 300 in EXMARaLDA eingegeben wurden. Die Transkripte werden zur systematischen Dokumentation mit Metadaten versehen und mit Schlagworten inhaltlich gegliedert. Diese Verschlagwortung der Texte mit etwa 40 thematischen Kategorien wie z. B. dem Schulbesuch, Kriegserlebnissen oder Motiven der landwirtschaftlichen Arbeit wird später neben der Stichwortsuche auch eine gezielte Abfrage von Textstellen zu den verschlagworteten Erzählthemen ermöglichen.

Die Projektmitarbeiterin Mirjam Nast ist mit der Umsetzung dieser Annotation betraut, welche das gesamte Korpus über die dialektologische Verwendung hinaus für die kulturwissenschaftliche Forschung nutzbar macht. Im Ergebnis liegen die alignierten Texte als interoperables xmlFile vor und können somit nachhaltig von jeder entsprechenden Datenbankumgebung sowohl auf ihre Metadaten als auch mithilfe von Stichworten auf Textinhalte durchsucht werden.

\footnotetext{
3 Aus der Beschreibung des Drittmittelprojekts Kultureller Wandel im Ländlichen Raum (LUI 2019b).
} 


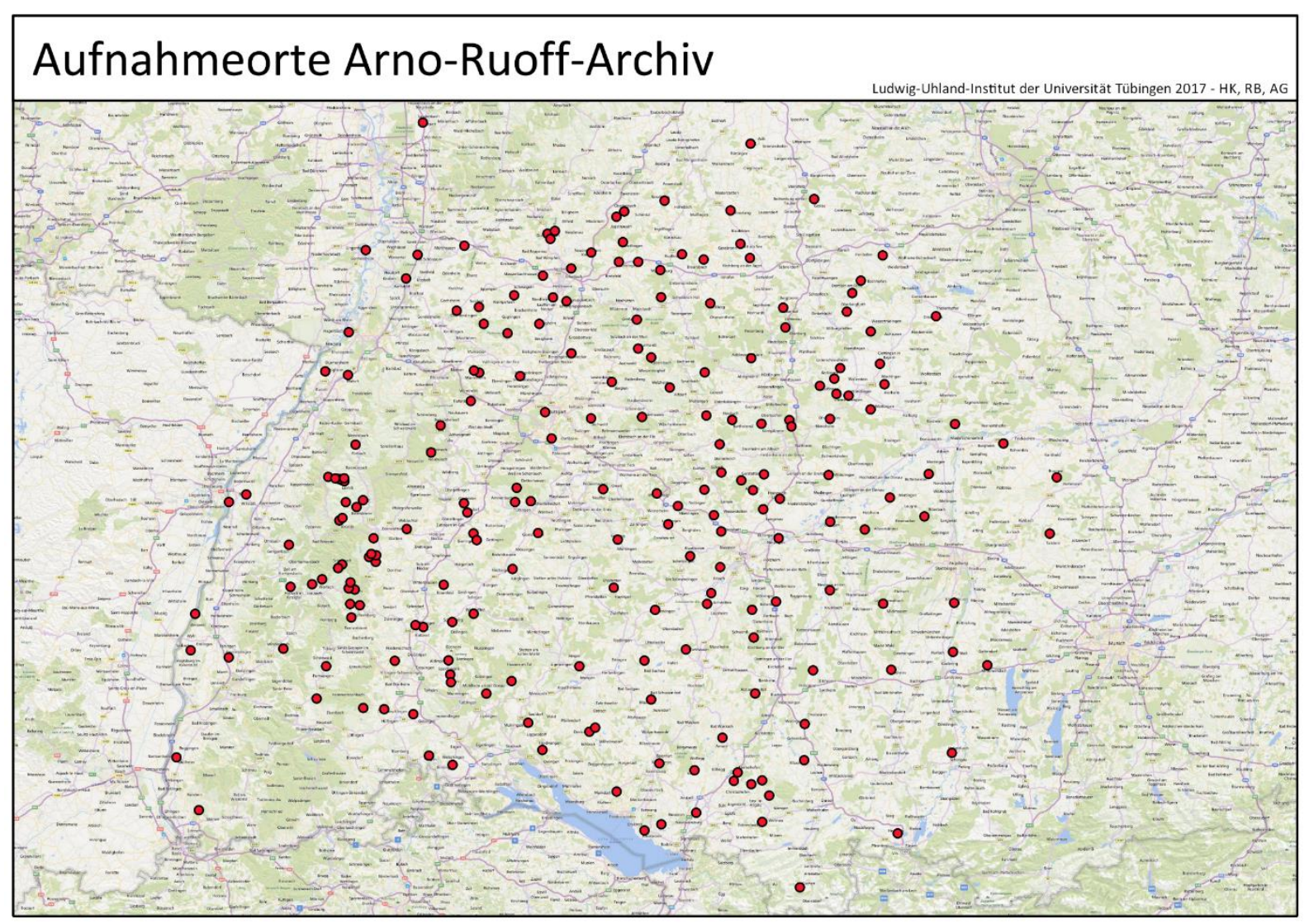

Abbildung 6: Die Aufnahmeorte des Arno-Ruoff-Archivs als georeferenzierte Ortspunkte

\section{Brand}

Im März 2017 wurde unsere Arbeit durch einen schweren Brand in unseren Tübinger Institutsräumen plötzlich unterbrochen. Sämtliche Arbeitsplätze, die gesamten im Verfügungsgebäude untergebrachten Bibliothekbestände des Ludwig-Uhland-Instituts inklusive zahlreicher nur noch antiquarisch erhältlicher Wörterbuchausgaben und große Teile des dort in der Biesingerstraße gelagerten Institutsarchivs wurden zerstört. Es mussten zunächst neue Arbeitsräume gefunden sowie die komplette Büroausstattung neu beschafft werden. Die Auswirkungen des Brandes in den Räumen des Arno-Ruoff-Archivs waren schließlich trotz des hohen materiellen Schadens und des enormen zeitlichen Verlustes in den Griff zu bekommen. Die umfassende, rasche und oft unbürokratische Unterstützung von allen Institutionen der Universität in Tübingen ermöglichte nach dreimonatiger Zwangspause doch eine Rückkehr zur Arbeit am Projekt. Mit dem Brand ist erneut deutlich geworden, wie wichtig die Digitalisierungsarbeit in den Archiven und die damit verbundene Möglichkeit zur dezentralen Lagerung von Beständen ist. Während durch den Brand viele Dutzend Regalmeter an Unterlagen des Archivs und der Projekte Sprachalltag I und II verloren gingen, überstanden die bereits digitalisierten Bestände den Brand unbeschadet auf ausgelagerten Servern.

Wir hoffen, dass wir bis zur endgültigen Unterbringung des Arno-Ruoff-Archivs in neuen Räumen weiterhin zügig mit der Arbeit vorankommen und bis zum Abschluss des Projekts auf der nächsten Alemannentagung alle gesteckten Ziele erreichen werden. 


\section{Literaturverzeichnis}

Baur, Gerhard W. (1967): Die Mundarten im nördlichen Schwarzwald. Textband, Kartenband. Marburg: Elwert. (=DDG 55a, b).

Bühler, Rudolf (2013): „Sprachalltag in Nord-Baden-Württemberg - Tradition und Zukunft bei der Erforschung gesprochener Sprache“. In: Alemannisches Jahrbuch 2011/2012. Freiburg i. Br., Alemannisches Institut: 209-230.

Bühler, Rudolf (2014): „Dialekte im Wandel““. In: Bühler, Rudolf/Bürkle, Rebekka/Leonhardt, Nina Kim (eds.): Sprachkultur - Regionalkultur. Neue Felder kulturwissenschaftlicher Dialektforschung. Tübingen, TVV: 241-254. (= Studien und Materialien des Ludwig-UhlandInstituts der Universität Tübingen 49).

Bühler, Rudolf (2016): Sprachwandeltendenzen in Baden-Württemberg. Eine diachrone Untersuchung am Beispiel der schwäbisch-fränkischen Dialektgrenze. Teil I: Textband. Teil II: Kartenband. http://dx.doi.org/10.15496/publikation-10682 [14.02.2019].

Bürkle, Rebekka (2014): „Sprachalltag in Unternehmen in Baden-Württemberg - Unternehmenskultur und individuelle Sprachpraxis“. In: Bühler, Rudolf/Bürkle, Rebekka/Leonhardt, Nina Kim (eds.): Sprachkultur - Regionalkultur. Neue Felder kulturwissenschaftlicher Dialektforschung. Tübingen, TVV: 136-153. (= Studien und Materialien des Ludwig-UhlandInstituts der Universität Tübingen 49).

Eisenmann, Fritz (1973): Die Satzkonjunktionen in gesprochener Sprache. Vorkommen und Funktion untersucht an Tonbandaufnahmen aus Baden-Württemberg, Bayrisch-Schwaben und Vorarlberg. Tübingen: Niemeyer. (= Idiomatica 2).

Gersbach, Bernhard/Graf, Rainer (1984, 1985): Wortbildung in gesprochener Sprache. Die Substantiv-, Verb-und Adjektiv-Zusammensetzungen und-Ableitungen im ,Häufigkeitswörterbuch gesprochener Sprache“. Band I und II. Tübingen: Niemeyer. (= Idiomatica 12 und 13).

Graf, Rainer (1977): Der Konjunktiv in gesprochener Sprache. Form, Vorkommen und Funktion untersucht an Tonbandaufnahmen aus Baden-Württemberg, Bayrisch-Schwaben, Vorarlberg und Liechtenstein. Tübingen: Niemeyer. (=Idiomatica 5).

Haag, Karl (1929/30): „Sprachwandel im Lichte der Mundartgrenzen (Mit 2 Karten: Grenzlandschaften des Schwäbischen gegen Fränkisch und Alemannisch)“. Teuthonista 6: 1-35.

Leonhardt, Nina Kim (2015): Dialektgrenzen als soziokulturelle Konstrukte. Subjektive Sprachräume in Nord-Baden-Württemberg. http://dx.doi.org/10.15496/publikation-89 [14.02.2019].

Löffelad, Peter (1989): Das Adjektiv in gesprochener Sprache. Gebrauch und Funktion untersucht an Tonbandaufnahmen südwestdeutscher Alltagssprache im Vergleich zu Tonbandaufnahmen der Sprache von Patienten im psychotherapeutischen Prozeß. Tübingen: Niemeyer. (= Idiomatica 16).

LUI (2019a): www.sprachalltag.de [16.09.2019].

LUI (2019b): https://uni-tuebingen.de/fakultaeten/wirtschafts-und-sozialwissenschaftlichefakultaet/faecher/fb-sozialwissenschaften/empirische-kulturwissenschaft/forschung/drittmittelprojekte/kultureller-wandel-im-laendlichen-raum/ [17.01.2019].

Mikosch, Ingrid (1987): Die Präpositionen in gesprochener Sprache. Vorkommen und Funktion untersucht an Tonbandaufnahmen aus Baden-Württemberg, Bayrisch-Schwaben und Vorarlberg. Tübingen: Niemeyer. (=Idiomatica 15). 
Moser, Hugo (1954/55): „Die schwäbisch-niederalemannische Sprachgrenze: Wandlung und Beharrung. Vorläufiges zur heutigen Lage. Mit 3 Karten“. Jahrbücher für Statistik und Landeskunde von Baden-Württemberg 1: 362-366.

Pfeffer, J. Alan/Lohnes, Walter F. W. (eds.) (1984): Grunddeutsch. Texte zur gesprochenen deutschen Gegenwartssprache. Überregionale Umgangssprache aus der Bundesrepublik Deutschland, der Deutschen Demokratischen Republik, Österreich und der Schweiz. Berlin/New York: de Gruyter. (= Phonai 28/30).

Ruoff, Arno (1965): „Wenkersätze auf Tonband?“. In: Sprachen - Zuordnung - Strukturen. Festgabe seiner Schüler für Eberhard Zwirner. Den Haag, Nijhoff: 94-113.

Ruoff, Arno (1967): „Mundart“. In: Staatliche Archivverwaltung Baden-Württemberg in Verbindung mit dem Landkreis Tübingen (eds.): Der Landkreis Tübingen. Amtliche Kreisbeschreibung. Band 1: Allgemeiner Teil. Stuttgart, Kohlhammer: 351-390.

Ruoff, Arno (1973): Grundlagen und Methoden der Untersuchung gesprochener Sprache. Einführung in die Reihe „Idiomatica“ mit einem Katalog der ausgewerteten Tonbandaufnahmen. Tübingen: Niemeyer. (=Idiomatica 1$)$.

Ruoff, Arno (1984): Alltagstexte I. Transkriptionen von Tonbandaufnahmen aus Baden-Württemberg und Bayrisch-Schwaben. Tübingen: Niemeyer. (=Idiomatica 10).

Ruoff, Arno (1985): Alltagstexte II. Transkriptionen von Tonbandaufnahmen aus Baden-Württemberg, Vorarlberg und Liechtenstein. Tübingen: Niemeyer. (=Idiomatica 11).

Ruoff, Arno (ed.) (1992): Die fränkisch-alemannische Sprachgrenze. Statik und Dynamik eines Übergangsgebiets untersucht und dargestellt in einem Projekt des Ludwig-Uhland-Instituts der Universität Tübingen. Textband. Tübingen: Niemeyer. (= Idiomatica 17/I).

Ruoff, Arno (1997): „Die Geschichte der Tübinger Arbeitsstelle ,Sprache in Südwestdeutschland“ 1955-1995. Mit einer Bibliographie“. In: Ruoff, Arno/Löffelad, Peter (eds.): Syntax und Stilistik der Alltagssprache. Beiträge der 12. Arbeitstagung zur alemannischen Dialektologie. 25. bis 29. September 1996 in Ellwangen/Jagst. Tübingen, Niemeyer: 283-296. (=Idiomatica 18).

Schallert (2014): Zur Syntax der Ersatzinfinitivkonstruktion. Typologie und Variation. Tübingen: Stauffenburg. (= Studien zur deutschen Grammatik 87).

Schmidt (2019): https://exmaralda.org/de/ueber-exmaralda/ [14.02.2019].

Schrambke, Renate (2009): „Sprache und Region. Der Südwestdeutsche Sprachatlas als Forschungsinstrument und Ausgangspunkt für vergleichende Detailstudien“. In: Keller-Drescher, Lioba/Tschofen, Bernhard (eds.): Dialekt und regionale Kulturforschung. Traditionen und Perspektiven einer Alltagssprachforschung in Südwestdeutschland. Tübingen, TVV: 67-97. (= Studien \& Materialien des Ludwig-Uhland-Instituts der Universität Tübingen 35).

Stadelmann, Manfred (1978): Orts- und Richtungsadverbien bei Siedlungsbezeichnungen. Verbreitung und Funktion in oberdeutschen Mundarten untersucht an Tonbandaufnahmen und Erhebungen der Tübinger Arbeitsstelle. Tübingen: Niemeyer. (=Idiomatica 6).

Streck, Tobias (2012): Phonologischer Wandel im Konsonantismus der alemannischen Dialekte Baden-Württembergs. Sprachatlasvergleich, Spontansprache und dialektometrische Studien. Stuttgart: Steiner. (=ZDL Beihefte 148).

Schwarz, Christian (2015): Phonologischer Dialektwandel in den alemannischen Basisdialekten Südwestdeutschlands im 20. Jahrhundert. Eine empirische Untersuchung zum Vokalismus. Stuttgart: Steiner. (=ZDL Beihefte 159). 
Tschofen, Bernhard/Keller-Drescher, Lioba (2009): „Traditionen und Perspektiven. Zur Neuvermessung eines alten Feldes“. In: Keller-Drescher, Lioba/Tschofen, Bernhard (eds.): Dialekt und regionale Kulturforschung. Traditionen und Perspektiven einer Alltagssprachenforschung in Südwestdeutschland. Tübingen, TVV: 9-18. (= Studien und Materialien des Ludwig-Uhland-Instituts der Universität Tübingen 35).

Vahl-Seyfarth, Ellen (1987): Das unbestimmte Subjekt in gesprochener Sprache. Vorkommen, Funktionen und Gebrauchsbedingungen untersucht an Tonbandaufnahmen aus BadenWürttemberg, Bayrisch-Schwaben und Vorarlberg. Tübingen: Niemeyer. (=Idiomatica 15).

Zwirner, Eberhard (1964): Anleitung zu sprachwissenschaftlichen Tonbandaufnahmen. Göttingen: Vandenhoeck \& Ruprecht. (= Lautbibliothek der deutschen Mundarten 31).

\section{Sprachatlanten}

Gabriel, Eugen et al. (eds.) (1989-2011): Südwestdeutscher Sprachatlas (SSA). Marburg: Elwert.

Klausmann, Hubert (ed.) (2015): Sprachatlas von Nord Baden-Württemberg. Band 1 - Kurzvokalismus. http://dx.doi.org/10.15496/publikation-7819 [14.02.2019].

Klausmann, Hubert (ed.) (2016): Sprachatlas von Nord Baden-Württemberg. Band 2: Langvokalismus und Diphthonge. Konsonantismus und Vokalquantitäten. http://dx.doi.org/10.15496/publikation-12899 [14.02.2019].

Klausmann, Hubert (ed.) (2017a): Sprachatlas von Nord Baden-Württemberg. Band 3 - Formengeographie. Kartenband. http://dx.doi.org/10.15496/publikation-20567 [14.02.2019].

Klausmann, Hubert (ed.) (2017b): Sprachatlas von Nord Baden-Württemberg. Band 3 - Formengeographie. Kommentarband. http://dx.doi.org/10.15496/publikation-20568 [14.02.2019].

Klausmann, Hubert (ed.) (2018a): Sprachatlas von Nord Baden-Württemberg (SNBW). Band I- Kurzvokalismus. Kartenband. 3. Auflage. http://dx.doi.org/10.15496/publikation-27775 [29.05.2019].

Klausmann, Hubert (ed.) (2018b): Sprachatlas von Nord Baden-Württemberg (SNBW). Band II - Langvokalismus, Diphthonge, Quantitäten, Konsonantismus. Kartenband. 2. Auflage. http://dx.doi.org/10.15496/publikation-27779 [29.05.2019].

Klausmann, Hubert (ed.) (2018c): Sprachatlas von Nord Baden-Württemberg (SNBW). Band III - Formengeographie. Kartenband. 2.Auflage. http://dx.doi.org/10.15496/publikation27780 [29.05.2019].

Klausmann, Hubert (ed.) (2019a): Sprachatlas von Nord Baden-Württemberg (SNBW). Band IV - Wortschatz 1. Kartenband. http://dx.doi.org/10.15496/publikation-27786 [29.05.2019]

Klausmann, Hubert (ed.) (2019b): Sprachatlas von Nord Baden-Württemberg (SNBW). Band $V$ - Wortschatz 2. Kartenband. http://dx.doi.org/10.15496/publikation-27792 [29.05.2019]. SBS (2013): www.bayerische-landesbibliothek-online.de/sprachatlas [14.02.2019]. 